\title{
Patrick Thaddeus
}

\section{A pioneer in the field of astrochemistry, Patrick Thaddeus discovered dozens of exotic molecules in space and helped revolutionize our view of the interstellar medium and star formation.}

P atrick Thaddeus did more than anyone else to demonstrate, as he was fond of saying, that chemistry was not a provincial subject that stopped five miles above our heads. As a pioneer in the field of astrochemistry, his elegant laboratory work provided ironclad identifications of hundreds of new molecules of astronomical interest, and his observational programme discovered about one-sixth of the 200 molecules known to exist in space. His early recognition that carbon monoxide would be an excellent tracer of the cold dense regions of space led directly to the discovery of giant molecular clouds and a revolution in our understanding of the interstellar medium and star formation.

Pat was born on 6 June 1932 in Arden, Delaware, and passed away peacefully on 28 April 2017 in Cambridge, Massachusetts. Apparently the small village of Arden did not provide enough of the rich intellectual stimulation that Pat would savour throughout his adult life, for as a youth he was known as a bit of a scamp. On seeing Pat's name in Scientific American years later, one of his teachers remarked that he would sooner have expected to see him in prison. His interests in instrumentation and astronomy were likely sparked by his decision as a teenager to take on the arduous task of building a reflecting telescope from scratch.

Pat obtained a bachelor's degree in physics from the University of Delaware in 1953, and a master's in theoretical physics as a Fulbright scholar at Oxford, UK, in 1955 . He was then taken on by Professor Charles Townes at Columbia University around the time Townes was completing his Nobel-prize-winning research on maser and laser emission. Pat's own thesis work, completed in 1960, was on the subject of beam maser spectroscopy.

Pat would spend most of the next 26 years in New York City, as a professor of physics at Columbia and, simultaneously, as a research physicist at the NASA Goddard Institute for Space Studies.

Following the discovery of the cosmic microwave background (CMB) in 1965 , Pat saw the opportunity to measure the $\mathrm{CMB}$ near the peak of its $3 \mathrm{~K}$ blackbody curve using the optical absorption lines

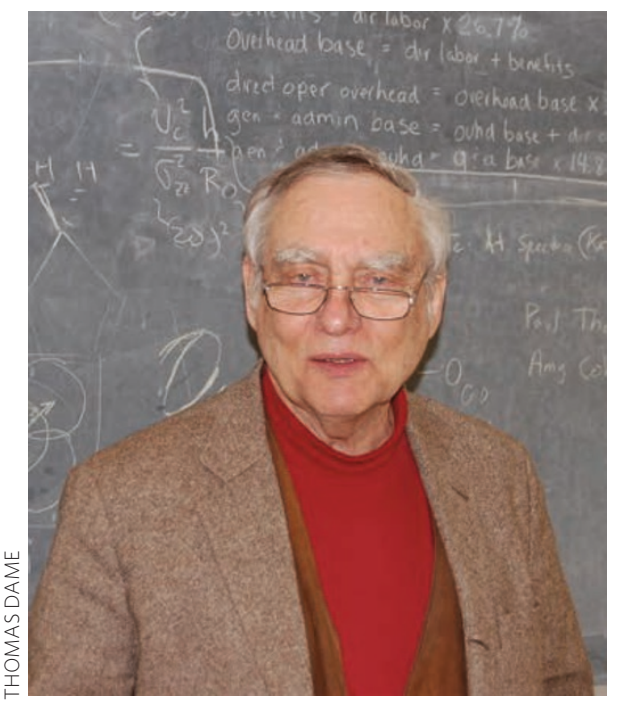

Patrick Thaddeus (1932-2017)

of the $\mathrm{CN}$ radical. With his prior optical experience limited to his handmade telescope, Pat relished learning the now arcane techniques of optical spectroscopy, the baking and cutting of glass plates to fit their curved holders, the careful guiding of the telescope for hours on end, the development of plates while immersed in total darkness and pungent odours. Based on over 100 hours of observations with the Lick 120-inch telescope, he and a student were able to measure a temperature of $2.78 \pm 0.10 \mathrm{~K}$ for the $\mathrm{CMB}$ at $2.6 \mathrm{~mm}$.

On 27 September 1974, Pat met in his office with John Mather, then his postdoc, Michael Hauser, David Wilkinson, and a few others to discuss the possibility of building a satellite to measure both the spectrum and possible spatial fluctuations of the CMB. This seminal meeting led directly to the COBE satellite project and a Nobel Prize for Mather. Ultimately, COBE's measurement of the $\mathrm{CMB}$ at $2.6 \mathrm{~mm}$ was within $0.6 \sigma$ of the value Pat had measured 18 years earlier using $\mathrm{CN}$.

After preliminary $\mathrm{CO}$ mapping with his students revealed that molecular regions in space were far larger than expected, Pat saw the need for a small but dedicated telescope to map these regions. In 1974 he and his group built a 4-foot telescope operating from a rooftop just a few hundred yards from Broadway. After over two decades of steady mapping with this instrument and a near-duplicate one that they installed in Chile in 1982, Pat and his students obtained what is still today the most extensive and widely used survey of the molecular Milky Way. More than 40 years later, both telescopes continue to yield important scientific results, including the discovery over the past decade of two new spiral arm features of the Galaxy. A total of $24 \mathrm{PhD}$ dissertations have been written based on observations or instrumental work with the two telescopes.

In 1986, Pat, along with several postdocs, students, and an engineer, loaded seven tractor trailers with laboratory equipment and their 4-foot telescope and headed north to the Center for Astrophysics (CfA), where he would work for another three decades as a professor of astronomy and applied physics at Harvard, and as a senior space scientist with the Smithsonian Astrophysical Observatory. The spectroscopy laboratory that he set up at the CfA has been one of the most productive in terms of scientific output and impact. Hundreds of exotic or 'non-terrestrial' molecules have been detected there, and nearly one-tenth of these were discovered in space based on highly precise laboratory frequencies. They include species such as $\mathrm{C}_{2} \mathrm{H}, \mathrm{C}_{4} \mathrm{H}, \mathrm{SiC}_{2}$, and cyclic $\mathrm{C}_{3} \mathrm{H}_{2}$, which are now widely used as chemical tracers of star formation. The spectacular discovery of negatively charged molecules in 2006 overturned conventional wisdom that such anions were too fragile to exist in space.

Pat Thaddeus will be remembered by his colleagues as much more than a brilliant scientist. As a teacher, he could illuminate the most obscure concepts with vivid analogies enabled by his own deep understanding. Hearing Pat give a scientific lecture, you felt as if he were grabbing you by the collar to convey his excitement. He was a superb politician in the best sense of the word. When grappling with a difficult problem, he would often dash to the phone to chat with the world's expert, invariably a close friend. He had very high standards for scientific exposition. Even now I 
feel a sense of unease that these words will be published without first passing below the vigorous red pen of Professor Thaddeus. He was a true intellectual with boundless interests. At a dinner with Pat, you were as likely to learn about the philosophy of quantum mechanics, the mushroom species around his Berkshire home, or the magnificent flight of the wandering albatross.

Pat Thaddeus loved what he did, and he was extremely good at it. In that sense he was a very fortunate person, as were we all for having known him.

\section{THOMAS DAME}

Thomas Dame is at the Harvard-Smithsonian Center for Astrophysics, 60 Garden Street, Cambridge, Massachusetts 02138, USA. He worked with Patrick Thaddeus for 40 years as a student, colleague and friend. e-mail:tdame@cfa.harvard.edu 